\title{
A new high-throughput method for simultaneous detection of drug resistance associated mutations in Plasmodium vivax dhfr, dhps and mdr1 genes
}

\author{
Céline Barnadas ${ }^{1,2 \dagger}$, David Kent ${ }^{3 \dagger}$, Lincoln Timinao', Jonah Iga', Laurie R Gray ${ }^{4}$, Peter Siba', Ivo Mueller ${ }^{1,2,5}$,
} Peter J Thomas ${ }^{3^{*}}$ and Peter A Zimmerman ${ }^{4^{*}}$

\begin{abstract}
Background: Reports of severe cases and increasing levels of drug resistance highlight the importance of improved Plasmodium vivax case management. Whereas monitoring $P$. vivax resistance to anti-malarial drug by in vivo and in vitro tests remain challenging, molecular markers of resistance represent a valuable tool for high-scale analysis and surveillance studies. A new high-throughput assay for detecting the most relevant markers related to P. vivax drug resistance was developed and assessed on Papua New Guinea (PNG) patient isolates.
\end{abstract}

Methods: Pvdhfr, pvdhps and pvmdr1 fragments were amplified by multiplex nested PCR. Then, PCR products were processed through an LDR-FMA (ligase detection reaction - fluorescent microsphere assay). 23 SNPs, including pvdhfr 57-58-61 and 173, pvdhps 382-383, 553, 647 and pvmdr1 976, were simultaneously screened in 366 PNG $P$. vivax samples.

Results: Genotyping was successful in $95.4 \%$ of the samples for at least one gene. The coexistence of multiple distinct haplotypes in the parasite population necessitated the introduction of a computer-assisted approach to data analysis. Whereas $73.1 \%$ of patients were infected with at least one wild-type genotype at codons 57,58 and 61 of pvdhfr, a triple mutant genotype was detected in $65.6 \%$ of the patients, often associated with the 117T mutation. Only one patient carried the $173 \mathrm{~L}$ mutation. The mutant 647P pvdhps genotype allele was approaching genetic fixation (99.3\%), whereas $35.1 \%$ of patients were infected with parasites carrying the pvmdr1 976F mutant allele.

Conclusions: The LDR-FMA described here allows a discriminant genotyping of resistance alleles in the pvdhfr, pvdhps, and pvmdr1 genes and can be used in large-scale surveillance studies.

\section{Background}

Plasmodium vivax is the most widespread of the malaria parasites infecting human hosts and may be responsible for up to 400 million infections each year $[1,2]$. The widespread belief that this parasite causes only benign malaria has been challenged recently by reports of severe pathology, including cerebral malaria, acute respiratory distress, acute renal failure, and severe

\footnotetext{
*Correspondence: pjthomas@case.edu; paz@case.edu

† Contributed equally

${ }^{3}$ Department of Mathematics, Case Western Reserve University, Cleveland USA

${ }^{4}$ Center for Global Health and Diseases, Case Western Reserve University, Cleveland, USA

Full list of author information is available at the end of the article
}

anaemia in patients with $P$. vivax infections [3-6]. In addition, there are increasing worldwide reports of treatment failure after administration of the standard drug regimens, mainly chloroquine (CQ) [7-15]. New drugs are now available, but they are significantly more costly than CQ plus primaquine, the standard treatment for vivax malaria for the last 50 years [16]. In order to change their treatment policies based on local evidence, countries need efficient methods to assess potential for resistance to current standard treatments.

Monitoring in vivo drug resistance remains challenging due to the ability of $P$. vivax to relapse from longlasting liver stages. At the same time, the recurrence of parasitaemia due to de novo infection can confound 
evaluation of treatment efficacy. Genotyping of recurrent infections [17] makes it possible to distinguish infection by parasites with different genotypes (new infections) $[18,19]$ from infection by parasites with identical genotypes (whether due to relapse or to recrudescence from blood-stage parasites that survived drug treatment) [20]. Of course, even molecular diagnosis cannot distinguish persistent infection from de novo infection by a genetically identical parasite. In vitro assays, which should provide drug susceptibility data free from the effects of confounding factors, such as host immunity, are still difficult to conduct because of the lack of stable, continuous $P$. vivax in vitro culture [5,21]. Molecular markers of resistance, therefore, represent a useful tool to monitor the introduction and spread of anti-malarial resistance. For $P$. vivax, these markers include mutations in genes encoding the dihydrofolate reductase (PvDHFR) and the dihydropteroate synthase (PvDHPS) that are involved in drug resistance to antifolates (pyrimethamine) [22], and sulphonamides (sulphadoxine) [23], respectively. The 976F mutation in the gene encoding the multidrug resistance 1 protein (PvMDR1), which has been associated with 4-aminoquinolines (amodiaquine, $\mathrm{CQ}$ ) resistance in some $[21,24]$ but not all studies $[21,25]$.

Further investigations are needed to assess the predictive value of molecular markers; their use in monitoring of $P$. vivax drug resistance needs to be addressed, geographically, at both a local and a large-scale level [26]. For these reasons, a new assay was developed to detect single nucleotide polymorphisms (SNPs) potentially associated with $P$. vivax drug resistance, in $p v d h f r$, pvdhps and pvmdr1 genes (total of 23 allelic variants). This post-PCR multiplex assay uses ligase detection reaction and fluorescent-microsphere technologies. A similar approach was previously developed to screen for Plasmodium falciparum drug resistance-associated SNPs [27]. The assay was validated using sequenced isolates and a subset of samples from Papua New Guinea (PNG), where CQ resistant $P$. vivax was first described in 1989 [12]. Resistance to sulphadoxine-pyrimethamine (SP) was observed to be low until 2000 [28], when PNG introduced $\mathrm{CQ}$ or amodiaquine plus SP as the national first line treatment for both $P$. falciparum and $P$. vivax. Since then, levels of resistance to this combination have increased [24,29]. Here the development and application of this multiplex assay is described; the frequency of $P$. vivax genetic markers associated with multiple drug resistance in PNG patient isolates is presented.

\section{Methods}

Study population and blood sample collection

Samples were collected between 2006 and 2007 through active detection of malaria infections in a cohort of children 1-4 yrs of age in Ilaita, East Sepik Province, Papua New Guinea [30]. The study was approved by the PNG Medical Research Advisory Council. Plasmodium vivax infected monkey blood (Sall and Thai-3 strains) was kindly provided by W. E. Collins (Centers for Disease Control and Prevention, Atlanta, GA). Plasmodium falciparum positive samples were obtained from a culture of the 3D7 strain.

\section{DNA template preparation}

DNA was extracted from cell pellets $(250 \mu \mathrm{L})$ using the QIAamp 96 DNA blood kit (Qiagen, Valencia, CA). Genomic DNA was extracted from $P$. vivax-infected monkey blood or from $P$. falciparum culture using the QIAamp DNA blood minikit (Qiagen, Valencia, CA).

\section{Plasmodium species diagnosis}

Plasmodium species diagnosis was performed using a post-PCR ligase detection reaction - fluorescent microsphere assay (LDR-FMA) as described previously $[30,31]$. Positive samples for P. vivax infections were identified and then assessed for mutations in $p v d h f r$, pvdhps and pvmdr1 genes.

\section{Multiplex PCR amplification of $P$. vivax dhfr, dhps and $m d r 1$ target sequences}

A nested PCR was developed to perform multiplex amplification of $P$. vivax dhfr, dhps and $m d r 1$ target sequences. All Nest 1 reactions were performed in a buffer containing 3 pmol of each primer, $67 \mathrm{mM}$ Tris$\mathrm{HCl}(\mathrm{pH}=8.8), 3.3 \mathrm{mM} \mathrm{MgSO} 4,16.6 \mathrm{mM}\left(\mathrm{NH}_{4}\right)_{2} \mathrm{SO}_{4}$, $10 \mathrm{mM}$ mercaptoethanol, $100 \mu \mathrm{M}$ (each) dATP, dGTP, dCTP, dTTP, and $2.5 \mathrm{U}$ of thermostable DNA polymerase. Nest 2 reactions were performed with $3 \mu \mathrm{L}$ of PCR products from the Nest 1 reaction using the same conditions except for the primers $(6 \mathrm{pmol})$. Primer sequences and size of PCR products are given in Table A1 (Additional file 1). PCR amplification was performed under the following conditions: Nest $1-94^{\circ} \mathrm{C}$ for $2 \mathrm{~min}$ utes and 35 cycles at $94^{\circ} \mathrm{C}$ for 30 seconds, $60^{\circ} \mathrm{C}$ for 30 seconds, $72^{\circ} \mathrm{C}$ for 3 minutes, and a final extension at $72^{\circ}$ $\mathrm{C}$ for 5 minutes; Nest $2-94^{\circ} \mathrm{C}$ for 2 minutes and 45 cycles at $94^{\circ} \mathrm{C}$ for 30 seconds, $63^{\circ} \mathrm{C}$ for 30 seconds, $72^{\circ} \mathrm{C}$ for 90 seconds, and a final extension at $72^{\circ} \mathrm{C}$ for $4 \mathrm{~min}$ utes. The specificity of the amplification was evaluated following electrophoresis on a $2 \%$ agarose gel in $1 \times \mathrm{TBE}$ buffer $(8.9 \mathrm{mM}$ Tris, $8.9 \mathrm{mM}$ boric acid, $2.0 \mathrm{mM}$ EDTA).

\section{Ligase detection reaction - fluorescence microspheres assay (LDR-FMA)}

A description of the three-step, post-PCR LDR-FMA, procedure has been provided in previous publications [27,31]. After PCR amplification of pvdhfr, pvdhps and 
pvmdr1 target sequences, products were subjected to a ligase detection reaction between modified upstream allele-specific and downstream conserved sequence primers. Modifications included the addition of unique TAG sequences at the 5 ' extremity of the upstream sequence-specific primers and 5' phosphorylation/3' biotinylation of the downstream primers. In a second step, hybridization occurred between TAG and anti-TAG oligonucleotide probes bound to fluorescent microspheres. The products of this reaction were then incubated in a solution containing streptavidin-phycoerythrin (SA-PE) to allow binding to the 3' biotin of the conserved sequence primer.

One $\mu \mathrm{L}$ of multiplex PCR product was subjected to the ligation reaction, performed in a solution $(15 \mu \mathrm{L})$ containing $20 \mathrm{mM}$ Tris- $\mathrm{HCl}$ buffer (pH 7.6), $25 \mathrm{mM}$ potassium acetate, $10 \mathrm{mM}$ magnesium acetate, $1 \mathrm{mM}$ $\mathrm{NAD}^{+}, 10 \mathrm{mM}$ dithiothreitol, 0.1\% Triton X-100, $10 \mathrm{nM}$ (200 fmol) each LDR probe, $1 \mu \mathrm{l}$ of each PCR product, and $2 \mathrm{U}$ of Taq DNA ligase (New England Biolabs, Beverly, MA). Reaction mixtures were initially heated to $95^{\circ}$ $\mathrm{C}$ for $1 \mathrm{~min}$, followed by 32 thermal cycles at $95^{\circ} \mathrm{C}$ for $15 \mathrm{~s}$ (denaturation) and $60.0^{\circ} \mathrm{C}$ for $2 \mathrm{~min}$ (annealing/ ligation). The multiplex LDR product $(5 \mu \mathrm{l})$ was then added to $60 \mu \mathrm{l}$ of hybridization solution (3 M tetramethylammonium chloride [TMAC], $50 \mathrm{mM}$ Tris- $\mathrm{HCl}$ [pH 8.0], 3 mM EDTA [pH 8.0], 0.10\% sodium dodecyl sulfate) containing 250 Luminex FlexMAPTM microspheres for each allelic set. Mixtures were heated to $95^{\circ}$ $\mathrm{C}$ for $90 \mathrm{~s}$ and incubated at $37^{\circ} \mathrm{C}$ for $40 \mathrm{~min}$ to allow hybridization between SNP-specific LDR products and microsphere-labelled anti-TAG probes. Following hybridization, $6 \mu \mathrm{l}$ of streptavidin-R-phycoerythrin (Molecular Probes, Eugene, OR) in TMAC hybridization solution $(20 \mathrm{ng} / \mu \mathrm{l})$ was added to the post-LDR mixture and incubated at $37^{\circ} \mathrm{C}$ for $40 \mathrm{~min}$ in Costar $6511 \mathrm{M}$ polycarbonate 96-well V-bottom plates (Corning Inc., Corning, NY). Detection of SNP-specific products (Classification signal $=$ microspheres fluorescence $/$ Reporter signal = SA-PE) was performed through a BioPlex liquid array reader (Bio-Rad laboratories, Hercules, CA). All fluorescence data were compiled into Microsoft Excel spreadsheets using Bio-Rad (Hercules, CA) software, Bio-Plex Manager 4.1.

Sequences of LDR primers are given in Table A2 (Additional file 2). These primers were designed to allow the detection of mutations at five pvdhfr codons (57-58-61, 117, 173), four pvdhps codons (382-383, 553, 647) and one pvmdr1 codon (976), with a total of 23 SNPs detected. LDR primers were designed according to available sequences published in the literature and sequences obtained from PNG isolates. To improve the specificity of the assay, a mismatch $(\mathrm{G} \rightarrow \mathrm{A})$ was introduced in the sequence of the primers (at nucleotide 178, accession no. X98123.1) hybridizing against codons 5758-61 of pvdhfr gene. This allowed a decrease of the allele-specific background observed.

\section{Obtaining controls and sequencing}

Pvdhfr, pvdhps and pvmdr1 genes were amplified by nested PCR from a subset of samples $(n=6)$; individual species-specific gene fragments were sequenced after purification using the QIAquick PCR purification kit (Qiagen, Valencia, CA). Additionally, six isolates (genotypes known) kindly provided by D. Ménard (Pasteur Institute, Madagascar) and C. Sibley (University of Washington, Seattle, WA) were amplified for $p v d h f r$ gene; PCR products were cloned to obtain positive controls for a selection of mutant pvdhfr genotypes: 57I (ata), 58R (agg, aga, cgt), 117N/117T (aac/acc), 173L (ctt). Three isolates were cloned in order to obtain pvdhps wild type, single mutant $383 \mathrm{G}$ and double mutant 382C-383G genotype controls, and two isolates to obtain pvmdr1 Y976 and 976F. Cloning was performed using a TOPO-TA vector cloning system, and Top10F competent cells (Invitrogen), according to the manufacturer's instructions; DNA was extracted using the QIAprep Spin Miniprep kit (Qiagen, Valencia, CA). Sequencing reactions were performed by single-pass bidirectional plasmid sequencing (Agencourt Biosciences, Beverly, MA).

\section{Results}

Plasmodium vivax infections were observed in 366 (52\%) of 704 samples evaluated by Plasmodium species diagnosis with a post-PCR LDR-FMA [31]; 113 samples (30.9\%) were co-infected with P. falciparum. A LDRFMA multiplex marker analysis was developed to detect SNPs in $p v d h f r, p v d h p s$ and $p v m d r 1$ genes related to anti-malarial drug resistance and used to genotype these 366 P. vivax samples.

\section{Validation of the nested multiplex PCR approach for a simultaneous amplification of pvdhfr, pvdhps and pvmdr 1 genes}

To improve the efficiency of the assay, a nested PCR was performed. Three sets of primers were combined in two-step multiplex PCR. Figure 1 shows the fragment sizes obtained (amplification of $P$. vivax Sal-I and Thai3 strains, and one field isolate), following agarose gel electrophoresis: $1423 \mathrm{bp}$ for $p v d h p s, 917$ bp for $p v d h f r$ and $545 \mathrm{bp}$ for pvmdr1. As mixed Plasmodium species infections are commonly observed in PNG, specificity of the amplification was checked using P. falciparum 3D7 genomic DNA. To evaluate the multiplex PCR amplification strategy, a subset of samples $(n=39)$ were analysed to determine if all three target sequences were amplified from all samples judged to be positive for $P$. 


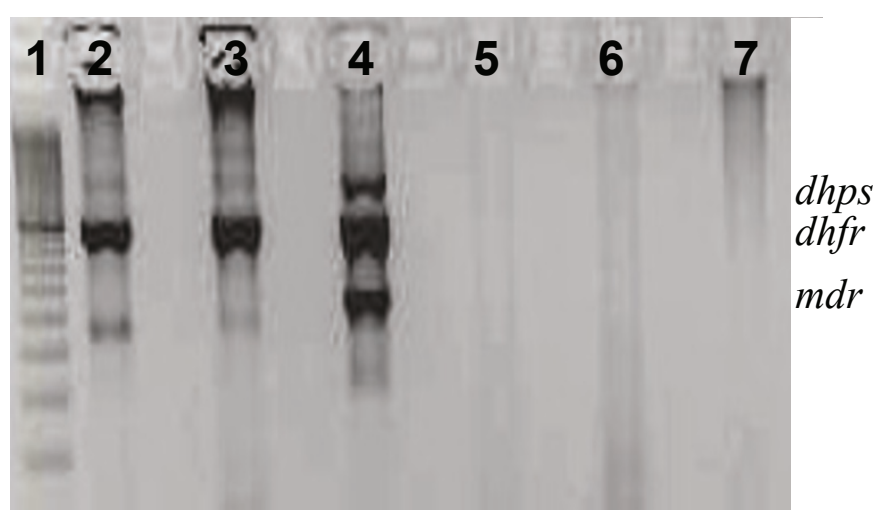

Figure $1 \mathrm{Gel}$ picture after nested multiplex amplification of pvdhfr, pvdhps and pvmdr1 fragments. 1: 100 bp DNA ladder. 2: P. vivax infected monkey blood (Thai 3). 3 P. vivax infected monkey blood (Sal1). 4: P. vivax positive PNG isolate. 5: P. falciparum 3d7. 6: Human DNA (Plasmodium sp negative). 7: Blank (water).

vivax by the species-specific diagnostic assay. From these samples, 37 (95\%) produced PCR products of the expected sizes for the three gene-specific target sequences using both single and multiplex PCR strategies.

\section{Validation of pvdhfr, pvdhps and pvmdr1 LDR-FMA probe specificity}

Sequenced isolates or clones were used to check the specificity of LDR product formation and fluorescent signals detected (Table A3, Additional file 3). At the time of this study, a control template for the pvdhps 553G and 647P mutant alleles was not available.

Eight distinct $p v d h f r$ haplotypes were discriminated across codons 57, 58 and 61 . Because of the proximity of the three codons, haplotype-specific primers covering the three codons and displaying the different allele combinations were designed. Results show that haplotype-specific background ranged from 168 to 3929 fluorescence units (FU); positive haplotype-specific signals ranged from 6968 to $25348 \mathrm{FU}$. Independently, three alleles were discriminated at codon 117, with allele-specific background ranging from 374 to 2740 FU, and positive allele-specific signals from 14942 to 24245 FU. Two alleles were discriminated at codon 173, with allele-specific background ranging from 566 to $8083 \mathrm{FU}$, and positive allele-specific signals from 19770 to 24564 FU.

Four pvdhps haplotypes were discriminated at codons 382 and 383 (using haplotype-specific primers). Results show that allele-specific background ranged from 202 to $1798 \mathrm{FU}$; positive allele-specific signals ranged from 4925 to $21682 \mathrm{FU}$. Two pvmdr1 alleles were discriminated at codon 976. Results show that allele-specific background ranged from 811 to 974 FU; positive allelespecific signals ranged from 15634 to $19634 \mathrm{FU}$.
Similar to what was described elsewhere [32], at some loci, an increase of the allele-specific background was observed proportionally to the intensity of another allele-specific signal (Figure 2). This effect was observed on $p v d h f r$ 57-58-61 FR(agg)T and pvdhfr 57-58-61 FR (aga) $\mathrm{T}$ allele-specific backgrounds (when $p v d h f r$ 57-5861 FST allele-specific signal was high), on pvdhfr 57-58$61 \mathrm{LR}(\mathrm{agg}) \mathrm{T}$ allele-specific background (when $p v d h f r$ 57-58-61 LR(aga)T allele-specific signal was high) and on pvdhfr 57-58-61 LR(agg)M allele-specific background (when pvdhfr 57-58-61 LR(aga)M allele-specific signal was high). A similar effect was observed on $p v d h f r$ 173L allele-specific background (when $p v d h f r$ I173 allele-specific signal was high). A more moderate effect was observed on pvdhps A647 allele-specific background (when pvdhps 647P allele-specific signal was high). Interpretation of the fluorescent signals using the polar/ multi-dimensional transformation of the data was thus performed.

\section{Computer-assisted multidimensional data analysis}

The coexistence of multiple distinct haplotypes in the parasite population necessitated the introduction of a novel approach to the analysis of LDR-FMA datasets from PNG.

Fluorescence signals from bi-allelic datasets were analysed using a polar transformation of the data in order to improve the data analysis of Plasmodium polyclonal infections, as previously described [32]. In the presence of one allele, an increase of the reciprocal allelic SNP signal can be observed, making direct determination of a constant threshold for positivity (presence of the second allele) inappropriate. Briefly, bivariate data were transformed from Cartesian coordinates $(\mathrm{x}$-axis $=$ allele $\mathrm{X}$ fluorescence signal; $\mathrm{y}$-axis $=$ allele $\mathrm{Y}$ fluorescence signal) into polar $(r, \theta)$ coordinates (here $r=\sqrt{x^{2}+y^{2}}$ and 


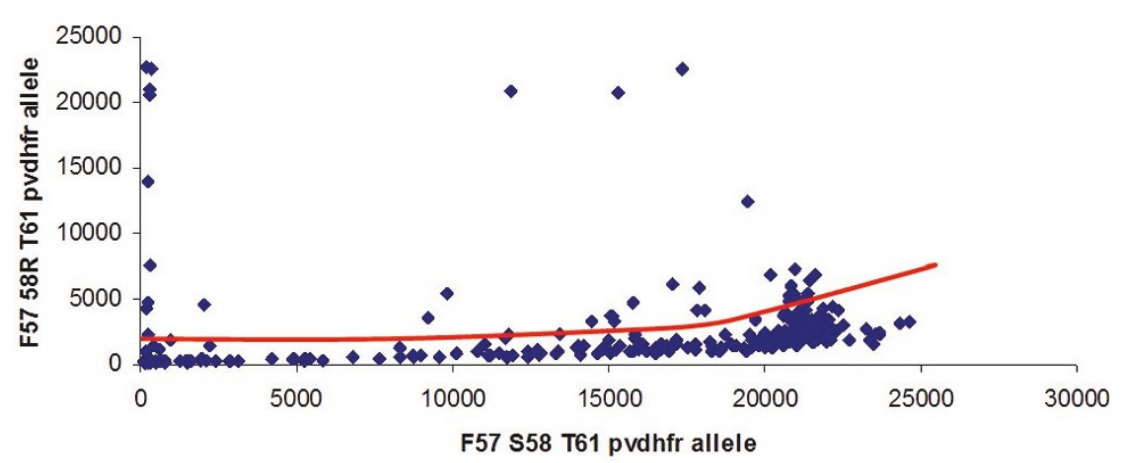

Figure 2 Increase of the allele-specific background (F57 58R T61 allele signal) observed proportionally to the intensity of another allele-specific signal (F57 S58 T61 allele signal).

$\theta=\arctan (y / x))$ to account for the approximately linear crosstalk interaction inducing the background signal. The magnitude of the combined signal, $r$, represents the distance in the plane between a point $(\mathrm{x}, \mathrm{y})$ and the origin of the graph. The angle, $\theta$, between the $\mathrm{x}$-axis and a ray extending from the origin to the point $(\mathrm{x}, \mathrm{y})$ represents the relative strength of the $\mathrm{y}$ versus the $\mathrm{X}$ signal. Samples with $\theta \approx 0$ correspond to pure infections of type $\mathrm{X}$, samples with $\theta \approx 90^{\circ}$ correspond to pure infections of type $\mathrm{Y}$, and intermediate values of $\theta$ representing samples with polyclonal infections. Subsequently, the threshold analysis included two steps. The first step determined a threshold for positivity for infections. The set of samples was divided into bins, from $r_{\min }$ to $r_{\max }$, each having a width of 100 fluorescence units (FU). The threshold of positivity was determined as the value of $r$ corresponding to the bin containing the first minimum (in number of counts) after the first maximum closest to $r=0$. In addition, we require that the number of samples in the bin be less than the number that would be expected under the uniform distribution (for a full technical description of the algorithm used for bi-allelic data please see [32]). The second step of the threshold analysis discriminated between polyclonal infections and allele-specific background signal. The set of samples was divided into 45 bins ranging from $\theta=0$ to $\theta=\pi / 2$ radians $\left(90^{\circ}\right)$. The threshold for the $\mathrm{X}+/ \mathrm{Y}-$ population was set at the first minimum in the histogram as $\theta$ increased past the local maximum close to $\theta=0\left(0^{\circ}\right.$, or horizontal). The threshold for the $\mathrm{X}-/ \mathrm{Y}+$ population was set at the first minimum in the histogram as $\theta$ decreased past the local maximum close to $\theta=\pi / 2\left(90^{\circ}\right.$, or vertical). Upon establishing the thresholds for distinguishing monoclonal versus polyclonal infections in the polar coordinate plane, an inverse polar coordinate transformation returned the data and the diagnosis threshold to the Cartesian plane.

Diagnosis of multi-allelic datasets (number of alleles > 2) was accomplished through a computer-assisted procedure analogous to the polar transformation introduced in DaRe et al [32]. Each sample was represented as a vector $\vec{x}_{i}=\left(x_{i 1}, \ldots, x_{i j}, \ldots, x_{i n}\right)$ where the first index $(i), 1 \leq i \leq N$, represented the sample number (out of $N$ samples) and the index $(j), 1 \leq j \leq n$ represented the allele (out of $n$ alleles). For example, the data set for pvdhfr locus 117 comprised $n=3$ alleles and $N=366$ samples; the data set for pvdhps loci 382-383 comprised $n=4$ alleles and $N=366$ samples; the data set for pvdhfr loci 57, 58 and 61 comprised $n=8$ alleles and $N$ $=366$ samples. The total magnitude of the fluorescence signal for sample $i$ was taken to be the Euclidean length of the vector, $L_{i}=\sqrt{\sum_{j=1}^{n} x_{i j}^{2}}$. A histogram was constructed of the $N$ values of $L_{i}$, using bins of width 100 fluorescence units, and the first minimum of the histogram occurring after the first maximum was used as a diagnostic cut-off for distinguishing infected versus uninfected samples. Figure 3 shows the histogram of fluorescence vector magnitudes for data from $p v d h f r$ 5758-61, along with the threshold obtained. For each sample with vector magnitude exceeding the diagnostic magnitude threshold, the quantities $\alpha_{i j}=x_{i j} / L_{i}$ were calculated. These values, known as direction cosines, equal the cosine of the angle $\theta_{i j}$ between the $i^{\text {th }}$ vector $\vec{x}_{i}$ and the $j^{\text {th }}$ coordinate axis in $n$-dimensional space. When the $i^{\text {th }}$ sample is infected by parasites carrying at least one allele, but not infected by parasites carrying the $j^{\text {th }}$ allele, the angle $\theta_{i j}$ is close to 90 degrees because the vector lies close to an (n-1)-dimensional subspace of the $n$-dimensional space spanned by the $(n-1)$ other vector components. Consequently, the vector component $x_{i j}$ is significantly smaller than those representing the alleles carried by infecting parasites, and the direction cosine $\alpha_{i j}=\cos \left(\theta_{i j}\right)$ will be close to zero. In order to assist the practitioner in calling alleles, our data analysis code (written in MATLAB) produces a histogram of all 


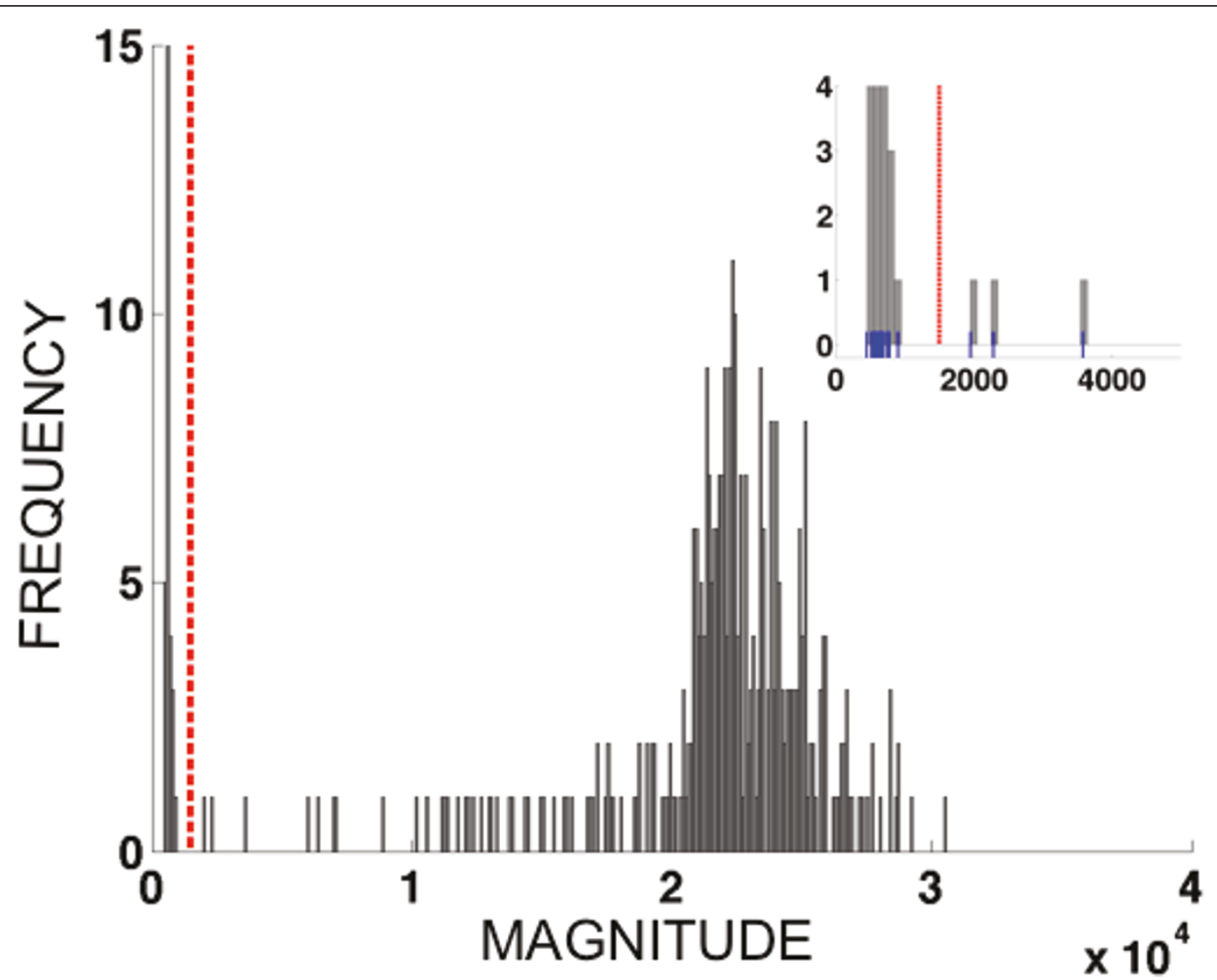

Figure 3 Histogram showing the distribution of fluorescence vector lengths $\left\{L_{i}: 1 \leq i \leq N\right\}$ for a multi-allelic data set (pvdhfr $57-58-61$, eight alleles). The dashed vertical line indicates the diagnosis threshold for infection vs. noninfection. Magnitude threshold $=1500 ; N_{\text {inf }}=338$. Histogram bin width $=100$ fluorescence units. Inset panel shows magnified region near the diagnostic threshold, including small vertical blue tick marks indicating the magnitudes for individual samples. Corresponding figures for pvdhps 382-383 (with four alleles) and for pvdhfr 117 (with three alleles) are available as Figures S1 and S3, respectively (See Additional Files 4 and 5).

$n \times N_{\text {inf }}$ values of the direction cosines for a given data set. Figure 4 shows the histogram of direction cosines produced for the $p v d h f r$ 57-58-61 data set $\left(n \times N_{\text {inf }}=8 \times\right.$ $338=2704$ values).

Once the analysis code produced a histogram, we inspected the histogram visually to identify appropriate diagnostic cut-off values for the direction cosines. Appropriate cut-offs were determined to be 0.20 for pvdhfr 57-58-61, 0.16 for pvdhps 382-383, and 0.24 for pvdhfr 117, see Figure 4, Additional files 4 and 5 (Figure S2 and S4), respectively. Heuristically, the direction cosine threshold was determined by identifying the maximum of the direction cosine histogram closest to $\alpha_{i j}=$ 0 , and $\alpha_{\text {thresh }}$, the location of the first minimum of the histogram below that maximum, as determined by eye, was used as the cut-off. Allele $j$ in sample $i$ was scored as positive if $\alpha_{i j}>\alpha_{\text {thresh }}$, and as negative if $\alpha_{i j} \leq \alpha_{\text {thresh }}$. Appropriate direction cosine thresholds for these data sets ranged over $0.16 \leq \alpha \leq 0.24$, corresponding to angles of approximately $75^{\circ}-80^{\circ}$. Please see Figures S1-S4
(Additional files 4 and 5) for additional examples of the magnitude and direction cosine histograms for multiallelic data sets.

\section{Application of the LDR-FMA to PNG $P$. vivax samples}

All three genes were successfully amplified and genotyped for 305 (83.3\%) of the isolates. A positive result for at least one gene associated with drug resistance ( $p v d h f r$, pvdhps or pvmdr1) was obtained for 349 (95.4\%) of the isolates in the first run. Of the isolates not successfully genotyped $90 \%$ showed lower positivity with the Plasmodium species diagnostic assay (500 to $10000 \mathrm{FU}$ ).

Based on $p v d h f r, p v d h p s$ and $p v m d r 1$ genotyping, a monoclonal infection was found in only 70/305 (23.0\%) samples. The multiplicity of infection (MOI) was determined based on the maximum number of alleles detected at one $p v d h f r$, pvdhps or pvmdr1 locus for each isolate and estimated at 2.05 with $p v d h f r$ displaying more allelic combinations $(\mathrm{MOI}=1.99)$ than $p v d h p s$ $(\mathrm{MOI}=1.03)$ and $p v m d r 1(\mathrm{MOI}=1.29)$. 


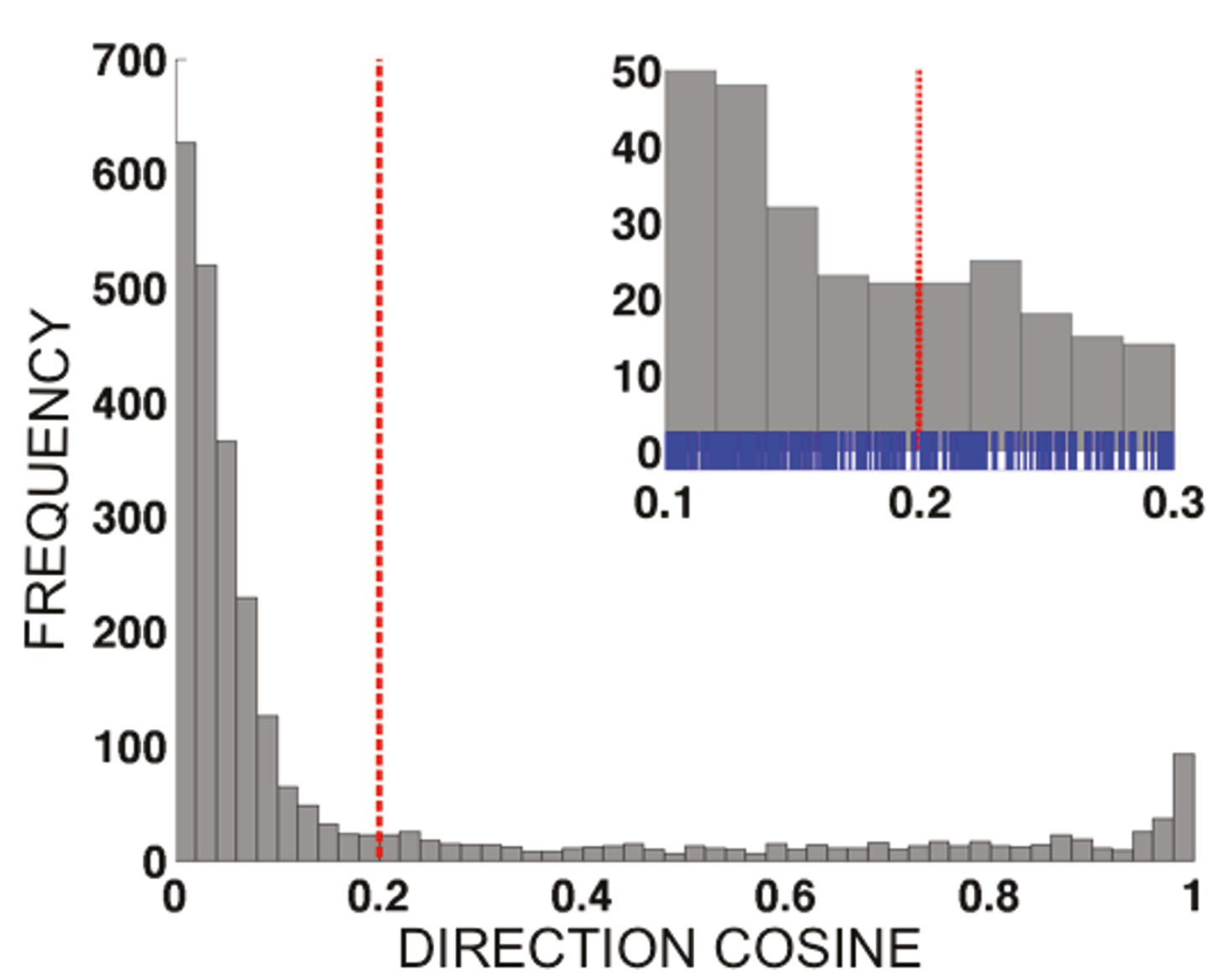

Figure 4 Histograms showing the distribution of all $N_{\text {inf }} \times n$ direction cosine values. $\left\{\alpha_{i j}: 1 \leq i \leq N_{\text {inf }}, 1 \leq j \leq n\right\}$ for different multi-allelic data sets. Only samples judged to be infected based on the magnitude histogram (see Figure 1) are included; the number of infected samples is denoted $N_{\text {inf }}$ and the number of alleles for a given data set is denoted $n$. The dashed vertical line indicates the diagnosis threshold for infection vs. noninfection of individual alleles. For the multi-allellic data sets, the thresholds were determined by visual inspection of the histograms. The pvdhfr 57-58-61 data contained eight alleles, giving $338 \times 8=2704$ direction cosines total in the histogram; direction cosine threshold $=0.2$. Histogram bin width $=0.02$. Inset panel shows magnified region near the diagnostic threshold, including small vertical blue tick marks indicating the direction cosines for individual alleles. Corresponding figures for pvdhps 382-383 (with four alleles) and for pvdhfr 117 (with three alleles) are available as Figures S2 and S4, respectively (See Additional Files 4 and 5).

Considering only the monoclonal infections $(\mathrm{n}=70)$, 37 isolates (52.9\%) displayed a wild-type $p v d h f r$ allele (F57-S58-T61-S117-I173). The quadruple mutant genotype (57L-58R-61-ㅡ-117T-I173) was found in 21 isolates (30.0\%); single $(\mathrm{n}=2 ; 2.9 \%)$, and double $(\mathrm{n}=10 ; 14.3 \%)$ mutant genotypes were also observed (Table 1). All but one of these samples displayed a single mutant type pvdhps 647P allele, and 12 of them (17.1\%) the pvmdr1 976F mutation.

When considering all infections (Table 2), the prevalence of multiple mutant SNP pvdhfr alleles was high. Of 305 infected patients, 223 (73.1\%) carried parasites with a wild-type genotype at codons 57,58 and 61 , whereas $200(65.6 \%)$ displayed at least a triple mutant genotype, most likely associated with the $117 \mathrm{~T}$ mutation, as this mutation was found in the isolates infecting $206(67.5 \%)$ patients. Only one patient carried parasites with the 173L mutation. Interestingly, 303 parasite isolates (99.3\%) carried the mutant pvdhps 647 P allele, approaching genetic fixation. In contrast, mutant allelic SNPs in codons 382 or 383 were found in the isolates infecting two to three (0.7 to $1 \%)$ patients. The mutant allele associated with $P$. vivax chloroquino-resistance, pvmdr1 976F, was observed in 107 (35.1\%) patient isolates.

\section{Discussion}

Here is described a new high-throughput method to detect SNPs associated with drug resistance of $P$. vivax, simultaneously in $p v d h f r, p v d h p s$ and $p v m d r 1$ genes. To improve the efficiency of this assay, a nested PCR approach was chosen which allowed a successful genotyping in $83.3 \%$ of the LDR-FMA $P$. vivax positive samples. 
Table 1 Pvdhfr, pvdhps, pvmdr1 genotypes prevalence in P. vivax monoclonal infections

\begin{tabular}{|c|c|}
\hline & Number of $P$. vivax monoinfected samples (\%) \\
\hline \multicolumn{2}{|c|}{ dhfr genotype (codons 57/58/61-117-173) } \\
\hline \multicolumn{2}{|l|}{ wild type: } \\
\hline FST-S-I & $37(52.9)$ \\
\hline \multicolumn{2}{|c|}{ double mutant type: } \\
\hline F $\underline{\mathbf{R}} T-\underline{\mathbf{N}}-\mathbf{I}$ & $2(2.9)$ \\
\hline LRT-S-I & $10(14.3)$ \\
\hline \multicolumn{2}{|c|}{ quadruple mutant type: } \\
\hline$\underline{\text { LRM-T-I }}$ & $21(30.0)$ \\
\hline \multicolumn{2}{|c|}{ dhps genotype (codons 382/383-553-647) } \\
\hline \multicolumn{2}{|c|}{ wild type: } \\
\hline SC-A-A & $1(1.4)$ \\
\hline \multicolumn{2}{|c|}{ single mutant type: } \\
\hline SC-A- $\underline{P}$ & 69 (98.6) \\
\hline \multicolumn{2}{|c|}{ mdr1 genotype (codon 976) } \\
\hline \multicolumn{2}{|c|}{ wild type: } \\
\hline Y & $58(82.9)$ \\
\hline \multicolumn{2}{|l|}{ mutant type: } \\
\hline$\underline{F}$ & $12(17.1)$ \\
\hline
\end{tabular}

The main challenge faced in developing this multiplexed assay was the interpretation of fluorescence data, due to the simultaneous screening of multiple alleles at one locus and the high level of polyclonality (up to four isolates infecting a single patient). As described previously [32], an increase of background (or off-target) fluorescent signal was consistently observed when PCR amplification produced strong gene-specific fragments. A polar transformation of the fluorescent data measured was performed for bi-allelic datasets; the usefulness of this transformation has been shown before [32]. In contrast, datasets with more than two alleles present required a multidimensional transformation of the data, based on the same principle as the polar transformation. This analysis was applied to three sets of multi-allelic data $(\mathrm{n}>2)$ : pvdhfr codons 57-58-61, pvdhfr 117, and pvdhps 382-383.

As in the case of bi-allelic data sets, while the coordinate transformation/histogram segmentation approach better accounted for advancing background due to offtarget hybridization, it is not a bulletproof algorithm that guarantees $100 \%$ accurate diagnosis. Indeed, given the intrinsic variability of any biological measurement process it is unlikely such a perfect algorithm exists. A significant limitation of the heuristic algorithm described here, at least from a theoretical point of view, is that independent determination of the diagnostic thresholds for each locus can lead to a situation in which a given sample is judged to be positively infected at some but not all loci, which is an inconsistent result. For example, the field data analysed here comprised 366 samples, each of which showed a positive diagnosis for $P$. vivax by LDR-FMA [31]. Of these samples, 17, or $5 \%$, were judged not to have enough net fluorescence signal to be considered positively infected based on the overall magnitude of the fluorescence vector for all seven loci interrogated. Of the 349 samples that showed a positive overall infection based on the magnitude histogram analysis, another $12.6 \%$ (44 samples) failed to have a positive single-allele diagnosis for any allele at one of the loci, leading to an incomplete haplotype determination. As a practical matter, in these cases the algorithm described under Methods was supplemented by censoring the samples with an incomplete haplotype determination.

A total of 23 alleles were screened simultaneously in the assay, with the possibility of extending the assay towards screening more alleles as 100 microspheres can be detected in the same multiplexed assay on the BioPlex liquid array reader. This high multiplexing capacity significantly reduces costs associated with diagnosing complex arrays of SNPs associated with anti-malarial drug resistance. While it would cost approximately 3 USD to genotype one sample using a real-time PCR assay screening for only four alleles (TaqMan), the costs of the PCR-LDR-FMA are similar to screen one sample for more than 20 alleles. In addition, as multiple loci of a gene are screened simultaneously, it is possible to identify new genotypes. If a positive signal is observed at one locus of the gene, but a negative signal at another, this would demonstrate the positivity of the PCR, and at the same time, the presence of a new allele not targeted by any of the LDR primers present in the reaction. In this case, it would then be necessary to sequence the 
Table 2 Pvdhfr, pvdhps, pvmdr1 genotypes identified in $305 P$. vivax infected patients

\begin{tabular}{|c|c|}
\hline & Number of infected patients (\%) \\
\hline \multicolumn{2}{|l|}{ dhfr genotype } \\
\hline \multicolumn{2}{|l|}{$57-58-61$} \\
\hline FST & $223(73.1)$ \\
\hline FRT & $28(9.2)$ \\
\hline$\underline{\mathrm{LRT}}$ & $116(38.0)$ \\
\hline$\underline{\text { LRM }}$ & $200(65.6)$ \\
\hline \multicolumn{2}{|l|}{117} \\
\hline S & $260(85.2)$ \\
\hline$\underline{\mathbf{N}}$ & $12(3.9)$ \\
\hline$\underline{T}$ & $206(67.5)$ \\
\hline \multicolumn{2}{|l|}{173} \\
\hline 1 & $304(99.7)$ \\
\hline L & $1(0.3)$ \\
\hline \multicolumn{2}{|l|}{ dhps genotype } \\
\hline \multicolumn{2}{|c|}{$382-383$} \\
\hline SC & $305(100)$ \\
\hline SE & $2(0.7)$ \\
\hline$\underline{\mathrm{C}} \mathrm{C}$ & $2(0.7)$ \\
\hline$\underline{\mathrm{CG}}$ & $3(1.0)$ \\
\hline \multicolumn{2}{|l|}{553} \\
\hline A & $305(100)$ \\
\hline \multicolumn{2}{|l|}{647} \\
\hline A & $5(1.6)$ \\
\hline$\underline{\mathbf{P}}$ & $303(99.3)$ \\
\hline \multicolumn{2}{|c|}{ mdr1 genotype } \\
\hline \multicolumn{2}{|c|}{976} \\
\hline Y & $287(94.1)$ \\
\hline$\underline{F}$ & $107(35.1)$ \\
\hline
\end{tabular}

PCR product in order to confirm the existence of a new genotype. Modifications to the LDR-FMA are then easily achieved by designing new sequence-specific primers.

Due to the wide use of sulphadoxine-pyrimethamine (SP) to treat falciparum malaria, $P$. vivax has been exposed worldwide to this anti-malarial drug. In Papua New Guinea, SP has been added to 4-aminoquinolines (CQ and amodiaquine) to treat $P$. falciparum and $P$. vivax malaria since 2000. It is therefore not surprising that $p v d h f r$ and $p v d h p s$ mutant alleles [23,33-36] were highly prevalent, with $65.6 \%$ of infected patients display-

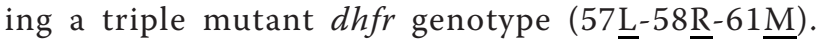
Because of a high rate of polyclonal infections $(83.3 \%)$ and a mean MOI of 1.99 calculated from pvdhfr genotyping results, it is difficult to reconstruct haplotypes and give more than an estimation of their prevalence. As shown by the results obtained from monoclonal infections, the $117 \underline{\mathrm{T}}$ mutation was associated with the triple mutant haplotype $57 \underline{L}-58 \underline{R}-61 \mathrm{M}$ in $100 \%$ of the cases. It is therefore possible to predict an overall rate of infection (among monoclonal and polyclonal infected patients) with a quadruple mutant $d h f r$ haplotype to occur in about $66 \%$ of the cases.

In vitro results (using a yeast model to express PvDHFR) have shown a strong correlation between mutations in $p v d h f r$ and the amount of drug needed to inhibit the growth in this system. The $50 \%$ inhibitory concentration (IC50) of pyrimethamine is increased by $100-460$ fold for a $d h f r$ double mutant $58 \mathrm{R}-117 \mathrm{~N}$, and more than 500-fold for a quadruple mutant enzyme $57 \underline{\mathrm{L}}-58 \underline{\mathrm{R}}-61 \underline{\mathrm{M}}-117 \underline{\mathrm{T}}$ in comparison to the IC50 measured for the wild-type enzyme [28,34]. Furthermore, the quadruple mutant haplotype has been associated with in vivo treatment failure after treatment with artesunate-SP in a study conducted in Papua, Indonesia [37]. Similarly, the quadruple mutant haplotype has been associated with SP treatment failure when compared to lower mutant parasites (triple mutant to wild type) [38]. The pvmdr1 Y976F mutation (when found in combination with $p v d h f r$ mutations) was associated with treatment failure in a PNG study conducted in 20042005 when $P$. vivax infected patients were treated with $\mathrm{AQ}+\mathrm{SP}[39]$.

Very few reports have been available on the prevalence of P. vivax dhfr and dhps mutations in PNG. From 19 isolates collected in 1998 in the Wosera (East Sepik Province) [28], only one (5.3\%) displayed a double 57L-58Rpvdhfr mutation. In the same study, of 25 isolates collected in 2000 in Liksul (Madang Province), nine displayed a $p v d h f r$ mutant genotype (six were double mutant $57 \underline{L}-58 \underline{R})$. More recently, genotyping results for 94 samples collected in three areas from PNG (Simbu, East Sepik and Madang provinces) between 2004 and 2005 showed higher prevalence of mutant genotypes and the appearance of quadruple mutant parasites (pvdhfr 57L-58R-61 $\underline{\mathrm{M}}-117 \underline{\mathrm{T}}$ ) in the population [24]. This increase is confirmed here with significantly higher rates of patients infected with mutant genotypes: $57 \mathrm{~L}$ was found in $78.4 \%$ of the participants, $58 \mathrm{R}$ in $83.0 \%, 61 \mathrm{M}$ in $65.9 \%, 117 \mathrm{~N} / \mathrm{T}$ in $70.8 \%$, versus, respectively, 59.6\%, 67.0\%, 20.2\% and $40.4 \%$ from the earlier study ( $\mathrm{p}<0.001$ ). Data from PNG isolates on $p v d h p s$ have not been generated; only one isolate from PNG was previously sequenced, showing a mutation at codon 647 (647) [40]. As for pvmdr1, the 976F mutation was found at a prevalence of $39.4 \%$ [24] and remains at the same level here (35.1\%) despite known high level of CQSP drug resistance in PNG (51.4\% after PCR correction [41]). It has already been discussed that 4-aminoquinoline resistance may result from a multigenic process involving different SNPs and/or gene amplification, 
variation in the level of expression $[24,25]$; it is therefore harder to correlate the $976 \mathrm{~F}$ mutation frequency with an increase of the in vivo level of drug resistance.

\section{Conclusions}

While Papua New Guinea and other malaria-endemic countries are changing their national malaria treatment policies, and implementing new treatment strategies, it will be important to assess the impact of such strategies on the evolution of $P$. vivax populations. The new highthroughput method described here represents a valuable tool that can be used for local or large-scale screening of $P$. vivax molecular markers of drug resistance.

\section{Additional material}

\section{Additional file 1: Table A1: Sequences of PCR primers}

Additional file 2: Table A2: Sequences of LDR primers for $P$. vivax dhfr, dhps and mdr1 genes

Additional file 3: Table A3: Fluorescence signals obtained for $P$. vivax samples (clones or sequenced isolates) of known dhfr, dhps and $m d r 1$ genotypes.

Additional file 4: Figures_S1S2. Figure S1: Magnitude histogram for dhps 382-383 (four alleles); magnitude threshold $=1500 ; N_{\text {inf }}=337$. Histogram bin width $=100$ fluorescence units. Figure S2: Direction cosine histogram for dhps 382-383 (four alleles, $337 \times 4=1348$ direction cosines total); direction cosine threshold $=0.16$. Histogram bin width $=0.02$. Figures S1-S2 show the magnitude and direction cosine histograms, respectively, for the multi-allelic data set dhps 382-383, which exhibited four positive alleles within the population. Inset panels show magnification of the region near the diagnostic threshold, including small vertical blue tick marks indicating the magnitudes of individual samples (S1) or values of direction cosines (S2). Compare Figures 1 and 2 of the main text.

Additional file 5: Figures_S3S4. Figure S3: Magnitude histogram for dhfr 117 (three alleles); magnitude threshold $=1000 ; N_{\text {inf }}=339$. Histogram bin width $=100$ fluorescence units. Figure S4: Direction cosine histogram for dhfr 117 (three alleles, $339 \times 3=1017$ direction cosines total); direction cosine threshold $=0.24$. Histogram bin width $=0.02$. Figures S3-S4 show the magnitude and direction cosine histograms, respectively, for multi-allelic data set $d$ fr 117 , which exhibited three positive alleles within the population. Compare Figures 1 and 2 of the main text.

\begin{abstract}
Acknowledgements and funding
We would like to thank Enmoore Lin, Benson Kiniboro, and all the field team members who helped collect the samples used in this analysis and Dr Richard Gray for his assistance in data analysis. We thank all the study participants. FINANCIAL SUPPORT: This study was supported by grants from the National Science Foundation (NSF-DMS-0720142, DUE-0634612, DMS1010434 and EF-1038677), the Fogarty International Center (TW007872 and TW007377), the Bill \& Melinda Gates Foundation, NHMRC (\#1010203), and Fondation Merieux.
\end{abstract}

\section{Author details}

'Vector Borne Diseases Unit, Papua New Guinea Institute of Medical Research (PNGIMR), Goroka, Papua New Guinea. ${ }^{2}$ Infection and Immunity Unit, Walter \& Eliza Hall Institute of Medical Research, Parkville, Australia. ${ }^{3}$ Department of Mathematics, Case Western Reserve University, Cleveland, USA. ${ }^{4}$ Center for Global Health and Diseases, Case Western Reserve University, Cleveland, USA. ${ }^{5}$ Centre de Recerca en Salut Internacional de Barcelona (CRESIB), Barcelona, Spain.

\section{Authors' contributions}

$\mathrm{CB}$ designed the assay, was responsible of laboratory work, and contributed to data analysis, and contributed to writing the paper. PJT and DK conceived the multi-allelic data analysis method, DK implemented it, and PJT and DK wrote the methods section on computer-assisted data analysis, and contributed to editing the manuscript. The results of the analysis were independently verified by CB and DK. LT and JI participated in the

laboratory work and in editing the manuscript. LG contributed to the design of the assay and editing the manuscript. IM and PS helped with data analysis and writing the paper. PAZ supervised laboratory work and the design of the assay, and contributed to data analysis and writing the paper. All authors read and approved the manuscript.

\section{Competing interests}

The authors declare that they have no competing interests.

Received: 20 April 2011 Accepted: 24 September 2011

Published: 24 September 2011

\section{References}

1. Guerra CA, Snow RW, Hay SI: Mapping the global extent of malaria in 2005. Trends Parasitol 2006, 22:353-358.

2. Hay SI, Guerra CA, Tatem AJ, Noor AM, Snow RW: The global distribution and population at risk of malaria: past, present, and future. Lancet Infect Dis 2004, 4:327-336.

3. Beg MA, Khan R, Baig SM, Gulzar Z, Hussain R, Smego RA Jr: Cerebral involvement in benign tertian malaria. Am J Trop Med Hyg 2002, 67:230-232.

4. Genton B, D'Acremont V, Rare L, Baea K, Reeder JC, Alpers MP, Muller I: Plasmodium vivax and mixed infections are associated with severe malaria in children: a prospective cohort study from Papua New Guinea. PLoS Med 2008, 5:e127.

5. Mueller I, Galinski MR, Baird JK, Carlton JM, Kochar DK, Alonso PL, del Portillo HA: Key gaps in the knowledge of Plasmodium vivax, a neglected human malaria parasite. Lancet Infect Dis 2009, 9:555-566.

6. Barcus MJ, Basri H, Picarima H, Manyakori C, Elyazar I, Bangs MJ, Maguire JD, Baird JK: Demographic risk factors for severe and fatal vivax and falciparum malaria among hospital admissions in northeastern Indonesian Papua. Am J Trop Med Hyg 2007, 77:984-991.

7. Baird JK, Basri H, Bangs MJ, Subianto B, Patchen LC, Hoffman SL: Resistance to chloroquine by Plasmodium vivax in Irian Jaya, Indonesia. Am J Trop Med Hyg 1991, 44:547-552.

8. Fryauff DJ, Tuti S, Mardi A, Masbar S, Patipelohi R, Leksana B, Kain KC, Bangs MJ, Richie TL, Baird JK: Chloroquine-resistant Plasmodium vivax in transmigration settlements of West Kalimantan, Indonesia. Am J Trop Med Hyg 1998, 59:513-518.

9. Kshirsagar NA, Gogtay NJ, Rajgor D, Dalvi SS, Wakde M: An unusual case of multidrug-resistant Plasmodium vivax malaria in Mumbai (Bombay), India. Ann Trop Med Parasitol 2000, 94:189-190.

10. Myat Phone K, Myint O, Myint L, Thaw Z, Kyin Hla A, Nwe Nwe Y: Emergence of chloroquine-resistant Plasmodium vivax in Myanmar (Burma). Trans R Soc Trop Med Hyg 1993, 87(6):687.

11. Phillips EJ, Keystone JS, Kain KC: Failure of combined chloroquine and high-dose primaquine therapy for Plasmodium vivax malaria acquired in Guyana, South America. Clin Infect Dis 1996, 23:1171-1173.

12. Rieckmann $\mathrm{KH}$, Davis DR, Hutton DC: Plasmodium vivax resistance to chloroquine? Lancet 1989, 2:1183-1184.

13. Ruebush TK, Zegarra J, Cairo J, Andersen EM, Green M, Pillai DR, Marquino W, Huilca M, Arevalo E, Garcia C, Solary L, Kain KC: Chloroquineresistant Plasmodium vivax malaria in Peru. Am J Trop Med Hyg 2003, 69:548-552.

14. Villalobos-Salcedo JM, Tada MS, Kimura E, Menezes MJ, Pereira da Silva LH In-vivo sensitivity of Plasmodium vivax isolates from Rondonia (western Amazon region, Brazil) to regimens including chloroquine and primaquine. Ann Trop Med Parasitol 2000, 94:749-758.

15. Alecrim MdG, Alecrim W, Macedo V: Plasmodium vivax resistance to chloroquine (R2) and mefloquine (R3) in Brazilian Amazon region. Rev Soc Bras Med Trop 1999, 32:67-68

16. Baird JK: Resistance to therapies for infection by Plasmodium vivax. Clinical Microbiol Rev 2009, 22:508-534. 
17. Koepfli C, Mueller I, Marfurt J, Goroti M, Sie A, Oa O, Genton B, Beck HP, Felger I: Evaluation of Plasmodium vivax genotyping markers for molecular monitoring in clinical trials. J Infect Dis 2009, 199:1074-1080.

18. Chen N, Auliff A, Rieckmann K, Gatton M, Cheng Q: Relapses of Plasmodium vivax infection result from clonal hypnozoites activated at predetermined intervals. J Infect Dis 2007, 195:934-941.

19. Imwong M, Snounou G, Pukrittayakamee S, Tanomsing N, Kim JR, Nandy A, Guthmann JP, Nosten F, Carlton J, Looareesuwan S, Nair S, Sudimack D, Day NP, Anderson TJ, White NJ: Relapses of Plasmodium vivax infection usually result from activation of heterologous hypnozoites. J Infect Dis 2007, 195:927-933.

20. Baird JK: Chloroquine resistance in Plasmodium vivax. Antimicrob Agents Chemother 2004, 48:4075-4083.

21. Suwanarusk R, Russell B, Chavchich M, Chalfein F, Kenangalem E, Kosaisavee V, Prasetyorini B, Piera KA, Barends M, Brockman A, Lek-Uthai U, Anstey NM, Tjitra E, Nosten F, Cheng Q, Price RN: Chloroquine resistant Plasmodium vivax: in vitro characterisation and association with molecular polymorphisms. PLOS ONE 2007, 2:e1089.

22. de Pecoulas PE, Tahar R, Ouatas T, Mazabraud A, Basco LK: Sequence variations in the Plasmodium vivax dihydrofolate reductase-thymidylate synthase gene and their relationship with pyrimethamine resistance. $\mathrm{Mol}$ Biochem Parasitol 1998, 92:265-273.

23. Imwong M, Pukrittayakamee S, Cheng Q, Moore C, Looareesuwan S, Snounou G, White NJ, Day NP: Limited polymorphism in the dihydropteroate synthetase gene (dhps) of Plasmodium vivax isolates from Thailand. Antimicrob Agents Chemother 2005, 49:4393-4395.

24. Marfurt J dMF, Brega S, Barbollat L, Müller I, Reeder JC, Beck HP, Picot S, Genton B: Molecular markers of in vivo Plasmodium vivax resistance to amodiaquine plus sulphadoxine-pyrimethamine: mutations in pvdhfr and pvmdr1. J Infect Dis 2008, 198:409-417.

25. Barnadas C, Ratsimbasoa A, Tichit M, Bouchier C, Jahevitra M, Picot S, Menard D: Plasmodium vivax resistance to chloroquine in Madagascar: clinical efficacy and polymorphisms in pvmdr1 and pvcrt-o genes. Antimicrob Agents Chemother 2008, 52:4233-4240.

26. Plowe CV, Roper C, Barnwell JW, Happi CT, Joshi HH, Mbacham W, Meshnick SR, Mugittu K, Naidoo I, Price RN, Shafer RW, Sibley CH, Sutherland CJ, Zimmerman PA, Rosenthal PJ: World Antimalarial Resistance Network (WARN) III: molecular markers for drug resistant malaria. Malar J 2007, 6:121.

27. Carnevale EP, Kouri D, DaRe JT, McNamara DT, Mueller I, Zimmerman PA: A multiplex ligase detection reaction-fluorescent microsphere assay for simultaneous detection of single nucleotide polymorphisms associated with Plasmodium falciparum drug resistance. J Clin Microbiol 2007, 45:752-761.

28. Hastings MD, Maguire JD, Bangs MJ, Zimmerman PA, Reeder JC, Baird JK, Sibley $\mathrm{CH}$ : Novel Plasmodium vivax dhfr alleles from the Indonesian Archipelago and Papua New Guinea: association with pyrimethamine resistance determined by a Saccharomyces cerevisiae expression system. Antimicrob Agents Chemother 2005, 49:733-740.

29. Karunajeewa HA, Mueller I, Senn M, Lin E, Law I, Gomorrai PS, Oa O, Griffin S, Kotab K, Suano P, Tarongka N, Ura A, Lautu D, Page-Sharp M, Wong R, Salman S, Siba P, llett KF, Davis TM: A trial of combination antimalarial therapies in children from Papua New Guinea. NEJM 2008, 359:2545-2557.

30. Lin E, Kiniboro B, Gray L, Dobbie S, Robinson L, Laumaea A, Schopflin S, Stanisic D, Betuela I, Blood-Zikursh M, Siba P, Felger I, Schofield L, Zimmerman P, Mueller I: Differential patterns of infection and disease with $P$. falciparum and $P$. vivax in young Papua New Guinean children. PloS One 2010, 5:e9047.

31. McNamara DT, Thomson JM, Kasehagen LJ, Zimmerman PA: Development of a multiplex PCR-ligase detection reaction assay for diagnosis of infection by the four parasite species causing malaria in humans. J Clin Microbiol 2004, 42:2403-2410.

32. DaRe JT, Kouri DP, Zimmerman PA, Thomas PJ: Differentiating Plasmodium falciparum alleles by transforming Cartesian $X, Y$ data to polar coordinates. BMC Genetics 2010, 11:57.

33. Barnadas C, Tichit M, Bouchier C, Ratsimbasoa A, Randrianasolo L, Raherinjafy R, Jahevitra M, Picot S, Menard D: Plasmodium vivax dhfr and dhps mutations in isolates from Madagascar and therapeutic response to sulphadoxine-pyrimethamine. Malar J 2008, 7:35.
34. Hawkins VN, Joshi H, Rungsihirunrat K, Na-Bangchang K, Sibley $\mathrm{CH}$ : Antifolates can have a role in the treatment of Plasmodium vivax. Trends Parasitol 2007, 23:213-222.

35. Imwong M, Pukrittayakamee S, Renia L, Letourneur F, Charlieu JP, Leartsakulpanich U, Looareesuwan S, White NJ, Snounou G: Novel point mutations in the dihydrofolate reductase gene of Plasmodium vivax: evidence for sequential selection by drug pressure. Antimicrob Agents Chemother 2003, 47:1514-1521.

36. Korsinczky M, Fischer K, Chen N, Baker J, Rieckmann K, Cheng Q: Sulfadoxine resistance in Plasmodium vivax is associated with a specific amino acid in dihydropteroate synthase at the putative sulfadoxinebinding site. Antimicrob Agents Chemother 2004, 48:2214-2222.

37. Tjitra E, Baker J, Suprianto S, Cheng Q, Anstey NM: Therapeutic efficacies of artesunate-sulfadoxine-pyrimethamine and chloroquine-sulfadoxinepyrimethamine in vivax malaria pilot studies: relationship to Plasmodium vivax dhfr mutations. Antimicrob Agents Chemother 2002, 46:3947-3953.

38. Hastings MD, Porter KM, Maguire JD, Susanti I, Kania W, Bangs MJ, Sibley $\mathrm{CH}$, Baird JK: Dihydrofolate reductase mutations in Plasmodium vivax from Indonesia and therapeutic response to sulfadoxine plus pyrimethamine. J Infect Dis 2004, 189:744-750.

39. Marfurt J, Mueller I, Sie A, Maku P, Goroti M, Reeder JC, Beck HP, Genton B: Low efficacy of amodiaquine or chloroquine plus sulfadoxinepyrimethamine against Plasmodium falciparum and $P$. vivax malaria in Papua New Guinea. Am J Trop Med Hyg 2007, 77:947-954.

40. Menegon M, Majori G, Severini C: Genetic variations of the Plasmodium vivax dihydropteroate synthase gene. Acta Trop 2006, 98:196-199.

41. Barnadas C, Koepfli C, Karunajeewa HA, Siba PM, Davis TM, Mueller I: Characterization of treatment failure in Plasmodium vivax efficacy trials by genotyping neutral and drug resistance associated markers. Antimicrob Agents Chemother 2011.

doi:10.1186/1475-2875-10-282

Cite this article as: Barnadas et al:: A new high-throughput method for simultaneous detection of drug resistance associated mutations in Plasmodium vivax dhfr, dhps and mdr1 genes. Malaria Journal 2011 10:282.

\section{Submit your next manuscript to BioMed Central and take full advantage of:}

- Convenient online submission

- Thorough peer review

- No space constraints or color figure charges

- Immediate publication on acceptance

- Inclusion in PubMed, CAS, Scopus and Google Scholar

- Research which is freely available for redistribution

Submit your manuscript at www.biomedcentral.com/submit
C Biomed Central 